\title{
Social distancing
}

Timothy C. Reluga

\section{Source}

Timothy C. Reluga. (2010). Game Theory of Social Distancing in Response to an

Epidemic. PLoS Comput Biol, vol. 6(5), e1000793. doi:10.1371/journal.pcbi.1000793.

Social distancing refers to the adoption of behaviors by individuals in a community that reduce those individuals' risk of becoming infected by limiting their contact with other individuals or reducing the transmission risk during each contact. 